$\xi=-1$

\title{
Fixed Point Theorems Under New Caristi Type Contraction in Bipolar Metric Space with Applications
}

\author{
B.Srinuvasa Rao ${ }^{1}$, G.N.V.Kishore ${ }^{1,2, *}$, S. Ramalingeswara Rao ${ }^{3}$ \\ ${ }^{I}$ Department of Mathematics, K L E F, Vaddeswaram, Guntur, Andhra Pradesh, India - 522502 \\ ${ }^{2,3}$ Department of Engineering Mathematics, S. R. K. R. Engineering College, Bhimavaram, Andhra Pradesh, India-534 204 \\ * Corresponding Author Email: kishore.apr2@gmail.com
}

\begin{abstract}
In this paper, the existence of fixed-point results in a complete bipolar metric spaces under new caristi type contraction is well established. Some attention gaining consequences are attained through our results. Finally, it presented an illustration which present applicability of the obtained results.
\end{abstract}

Keywords: Bipolar metric space; covariant map; fixed point; lower semi continuous function; new caristi type contraction.

\section{Introduction and Preliminaries}

Fixed point theory plays a vital role in applications of many branches of mathematics. Finding fixed points of generalized contraction mappings has become the focus of well-built research activity in fixed point theory. Recently, many investigators have published various papers on fixed point theory and applications in different ways. One of the recently popular topics in fixed point theory is to cast the existence of fixed points of contraction mappings in bipolar metric spaces which can be considered as generalizations of Banach contraction principle. In 2016, Mutlu and Gürdal [1] initiated the concepts of bipolar metric space and they also investigated some fixed point and coupled fixed point results on this space ([1], [2]). Caristi's fixed point theorem [3] is a renowned extension of Banach contraction principle [4]. The proof of caristi's results has been generalized and extended in many directions see ([5] - [13]). In this paper, we shall establish some fixed point results for covariant mapping under different new caristi type contractive conditions. We have illustrated the validity of the hypotheses of our results. First we recall some basic definitions and results.

Definition 1.1: [1] Let $U$ and V be a two non-empty sets. Suppose that $\mathrm{d}: \mathrm{U} \times \mathrm{V} \rightarrow[0, \infty)$ be a mapping satisfying the below properties:

(i) If $d(u, v)=0$, then $u=v$ for all $(u, v) \in U \times V$,

(ii) If $u=v$, then $d(u, v)=0$, for all $(u, v) \in U \times V$,

(iii) If $\mathrm{d}(\mathrm{u}, \mathrm{v})=\mathrm{d}(\mathrm{v}, \mathrm{u})$, for all $\mathrm{u}, \mathrm{v} \in \mathrm{U} \cap \mathrm{V}$

(iv) If $\mathrm{d}\left(u_{1}, v_{2}\right) \leq \mathrm{d}\left(u_{1}, v_{1}\right)+\mathrm{d}\left(u_{2}, v_{1}\right)+\mathrm{d}\left(u_{2}, v_{2}\right)$ for all $u_{1}$, $u_{2} \in \mathrm{U}$, and $v_{1}, v_{2} \in \mathrm{V}$.

Then the mapping $d$ is termed as Bipolar-metric of the pair $(U, V)$ and the triple $(\mathrm{U}, \mathrm{V}, \mathrm{d})$ is termed as Bipolar-metric space.

Definition 1.2: [1] Assume $\left(U_{1}, V_{1}\right)$ and $\left(U_{2}, V_{2}\right)$ as two pairs of sets and a function as F: $U_{1} \cup V_{1} \rightrightarrows U_{2} \cup V_{2}$ is said to be a covariant map. If $\mathrm{F}\left(U_{1}\right) \subseteq U_{2}$ and $\mathrm{F}\left(V_{1}\right) \subseteq V_{2}$ and denote this with $\mathrm{S}$ : $\left(A_{1}, B_{1}\right) \rightrightarrows\left(A_{2}, B_{2}\right)$. And the mapping $\mathrm{S}: U_{1} \cup V_{1} \rightleftarrows U_{2} \cup V_{2}$ is said to be a contravariant map. If $\mathrm{F}\left(U_{1}\right) \subseteq V_{2}$, and $\mathrm{F}\left(V_{1}\right) \subseteq U_{2}$, and write F: $\left(U_{1}, V_{1}\right) \rightleftarrows\left(U_{2}, V_{2}\right)$. In particular, if $d_{1}$ and $d_{2}$ are bipolar metric on $\left(U_{1}, V_{1}\right)$ and $\left(U_{2}, V_{2}\right)$, respectively, we sometimes use the notation F: $\left(U_{1}, V_{1}, d_{1}\right) \rightrightarrows\left(U_{2}, V_{2}, d_{2}\right)$ and F: $\left(U_{1}, V_{1}, d_{1}\right) \rightleftarrows\left(U_{2}, V_{2}, d_{2}\right)$.

Definition 1.3: [1] Assume (U, V, d) as a bipolar metric space. A point $\mathrm{v} \in \mathrm{U} U \mathrm{~V}$ is termed as a left point if $\mathrm{v} \in \mathrm{U}$, a right point if $\mathrm{v}$ $\in \mathrm{V}$ and a central point if both. Similarly, a sequence $\left\{u_{n}\right\}$ on the set $\mathrm{U}$ and a sequence $\left\{v_{n}\right\}$ on the set $\mathrm{V}$ are called a left sequence and right sequence respectively. In a bipolar metric space, sequence is the simple term for a left or right sequence. A sequence $\left\{v_{n}\right\}$ is considered convergent to a point $\mathrm{v}$, if and only if $\left\{v_{n}\right\}$ is the left sequence, $\mathrm{v}$ is the right point and $\lim _{n \rightarrow \infty} \mathrm{d}\left(v_{n}, \mathrm{v}\right)=0$; or $\left\{v_{n}\right\}$ is a right sequence, $\mathrm{v}$ is a left point and $\lim _{n \rightarrow \infty} \mathrm{d}\left(\mathrm{v}, v_{n}\right)=0$. A bi-sequence $\left(\left\{u_{n}\right\},\left\{v_{n}\right\}\right)$ on $(\mathrm{U}, \mathrm{V}, \mathrm{d})$ is a sequence on the set $\mathrm{U} \times \mathrm{V}$. If the sequence $\left\{u_{n}\right\}$ and $\left\{v_{n}\right\}$ are convergent, then the bisequence $\left(\left\{u_{n}\right\},\left\{v_{n}\right\}\right)$ is said to be convergent. $\left(\left\{u_{n}\right\},\left\{v_{n}\right\}\right)$ is Cauchy sequence, if $\lim _{n \rightarrow \infty} \mathrm{d}\left(u_{n}, v_{n}\right)=0$. In a bipolar metric space, every convergent Cauchy bi-sequence is bi-convergent. A bipolar metric space is called complete, if every Cauchy bisequence is convergent hence bi-convergent.

Definition 1.4: [1] Let $\left(U_{1}, V_{1}, d_{1}\right)$ and $\left(U_{2}, V_{2}, d_{2}\right)$ be a bipolar metric spaces.

(i) $\mathrm{A}$ map $\mathrm{F}$ is called continuous, if it left continuous at each point $\mathrm{u} \in U_{1}$ and right continuous at each point $\mathrm{v} \in V_{1}$

(ii) A contravariant map F: $\left(U_{1}, V_{1}, d_{1}\right) \Rightarrow\left(U_{2}, V_{2}, d_{2}\right)$ is continuous if and only if it is continuous as a covariant map F: $\left(U_{1}, V_{1}, d_{1}\right) \rightrightarrows\left(U_{2}, V_{2}, d_{2}\right)$.

It can be seen from the definition (1.3) that a covariant or a contravariant map F: $\left(\boldsymbol{U}_{1}, \boldsymbol{V}_{\mathbf{1}}, \boldsymbol{d}_{\mathbf{1}}\right) \rightrightarrows\left(\boldsymbol{U}_{2}, \boldsymbol{V}_{\mathbf{2}}, \boldsymbol{d}_{\mathbf{2}}\right)$ is continuous if and only if $\left(\boldsymbol{u}_{\boldsymbol{n}}\right) \rightarrow \boldsymbol{v}$ on $\left(\boldsymbol{U}_{\mathbf{1}}, \boldsymbol{V}_{\mathbf{1}}, \boldsymbol{d}_{\mathbf{1}}\right)$ implies $\mathrm{F}\left(\left(\boldsymbol{u}_{n}\right)\right) \rightarrow \boldsymbol{F}(\boldsymbol{v})$ on $\left(\boldsymbol{U}_{2}, \boldsymbol{V}_{2}, \boldsymbol{d}_{2}\right)$

\section{Main Results}

In this section, we give some fixed point theorems for covariant mapping satisfying various new caristi type contractive conditions in bipolar metric spaces. 
Theorem 2.1: Let $(\mathrm{U}, \mathrm{V}, \mathrm{d})$ be a complete bipolar metric spaces, suppose that $\mathrm{F}:(\mathrm{U}, \mathrm{V}) \rightrightarrows(\mathrm{U}, \mathrm{V})$ be a covariant mapping satisfies

$\mathrm{d}(\mathrm{Fa}, \mathrm{Fb}) \leq \psi(\boldsymbol{\alpha}(\boldsymbol{a})) \boldsymbol{\alpha}(\mathrm{a})-\boldsymbol{\alpha}(\mathrm{Fa})+\psi(\boldsymbol{\beta}(\boldsymbol{b})) \boldsymbol{\beta}(\mathrm{b})-\boldsymbol{\beta}(\mathrm{Fb})$

for all $\mathrm{a} \in \boldsymbol{U}, \mathrm{b} \in \boldsymbol{V}$, where $\boldsymbol{\alpha}, \boldsymbol{\beta}: \mathrm{U} \cup \mathbf{V} \rightarrow[0, \infty)$ are lower semi continuous functions and $\psi:(-\infty, \infty) \rightarrow(0,1)$ be a continuous function and provided that $\mathrm{F}$ is continuous. Then the mapping F:UU $\boldsymbol{V} \rightarrow \boldsymbol{U} \cup \boldsymbol{V}$ has a unique fixed point.

Proof: Let $\boldsymbol{a}_{\mathbf{0}} \in \mathrm{U}$ and $\boldsymbol{b}_{\mathbf{0}} \in \mathrm{V}$, we construct the bisequence $\left(\left\{\mathbf{a}_{\mathbf{n}}\right\},\left\{\mathbf{b}_{\mathbf{n}}\right\}\right)$ in $(\mathrm{U}, \mathrm{V})$ as $\mathrm{F} \boldsymbol{a}_{\boldsymbol{n}}=\boldsymbol{a}_{\boldsymbol{n}+\mathbf{1}}$ and $\mathrm{F} \boldsymbol{b}_{\boldsymbol{n}}=\boldsymbol{b}_{\boldsymbol{n}+\mathbf{1}}$ for $\mathrm{n}=0,1$, $2, \cdots \cdots$

By using the (1), we have

$$
\begin{array}{r}
\mathrm{d}\left(\boldsymbol{a}_{n}, \boldsymbol{b}_{n}\right)=\mathrm{d}\left(\boldsymbol{F} \boldsymbol{a}_{n-1}, \boldsymbol{F} \boldsymbol{b}_{n-1}\right) \\
\leq \psi\left(\boldsymbol{\alpha}\left(\boldsymbol{a}_{n-1}\right)\right) \boldsymbol{\alpha}\left(\boldsymbol{a}_{n-1}\right)-\boldsymbol{\alpha}\left(\mathrm{F} \boldsymbol{a}_{n-1}\right) \\
+\psi\left(\boldsymbol{\beta}\left(\boldsymbol{b}_{n-1}\right)\right) \boldsymbol{\beta}\left(\boldsymbol{b}_{n-1}\right)-\boldsymbol{\beta}\left(\mathrm{F} \boldsymbol{b}_{n-1}\right) \\
\leq \psi\left(\boldsymbol{\alpha}\left(\boldsymbol{a}_{n-1}\right)\right) \boldsymbol{\alpha}\left(\boldsymbol{a}_{n-1}\right)-\boldsymbol{\alpha}\left(\boldsymbol{a}_{n}\right) \\
+\psi\left(\boldsymbol{\beta}\left(\boldsymbol{b}_{n-1}\right)\right) \boldsymbol{\beta}\left(\boldsymbol{b}_{n-1}\right)-\boldsymbol{\beta}\left(\boldsymbol{b}_{n}\right) \\
\leq \boldsymbol{\alpha}\left(\boldsymbol{a}_{n-1}\right)-\boldsymbol{\alpha}\left(\boldsymbol{a}_{n}\right)+\boldsymbol{\beta}\left(\boldsymbol{b}_{n-1}\right)-\boldsymbol{\beta}\left(\boldsymbol{b}_{n}\right) \\
\text { and } \boldsymbol{\alpha}\left(\boldsymbol{a}_{n}\right)+\boldsymbol{\beta}\left(\boldsymbol{b}_{n}\right) \leq \psi\left(\boldsymbol{\alpha}\left(\boldsymbol{a}_{n-1}\right)\right) \boldsymbol{\alpha}\left(\boldsymbol{a}_{n-1}\right) \\
\quad+\psi\left(\boldsymbol{\beta}\left(\boldsymbol{b}_{n-1}\right)\right) \boldsymbol{\beta}\left(\boldsymbol{b}_{n-1}\right) \\
<\boldsymbol{\alpha}\left(\boldsymbol{a}_{n-1}\right)+\boldsymbol{\beta}\left(\boldsymbol{b}_{n-1}\right)
\end{array}
$$

and also

$$
\begin{array}{r}
\mathrm{d}\left(\boldsymbol{a}_{n}, \boldsymbol{b}_{n+1}\right)=\mathrm{d}\left(\boldsymbol{F} \boldsymbol{a}_{n-1}, \boldsymbol{F} \boldsymbol{b}_{n}\right) \\
\leq \psi\left(\boldsymbol{\alpha}\left(\boldsymbol{a}_{n-1}\right)\right) \boldsymbol{\alpha}\left(\boldsymbol{a}_{n-1}\right)-\boldsymbol{\alpha}\left(\mathrm{F} \boldsymbol{a}_{n-1}\right) \\
+\psi\left(\boldsymbol{\beta}\left(\boldsymbol{b}_{n}\right)\right) \boldsymbol{\beta}\left(\boldsymbol{b}_{n}\right)-\boldsymbol{\beta}\left(\mathrm{F} \boldsymbol{b}_{n}\right) \\
\leq \psi\left(\boldsymbol{\alpha}\left(\boldsymbol{a}_{n-1}\right)\right) \boldsymbol{\alpha}\left(\boldsymbol{a}_{n-1}\right)-\boldsymbol{\alpha}\left(\boldsymbol{a}_{n}\right) \\
+\psi\left(\boldsymbol{\beta}\left(\boldsymbol{b}_{n}\right)\right) \boldsymbol{\beta}\left(\boldsymbol{b}_{n}\right)-\boldsymbol{\beta}\left(\boldsymbol{b}_{n+1}\right) \\
\leq \boldsymbol{\alpha}\left(\boldsymbol{a}_{n-1}\right)-\boldsymbol{\alpha}\left(\boldsymbol{a}_{n}\right)+\boldsymbol{\beta}\left(\boldsymbol{b}_{n}\right)-\boldsymbol{\beta}\left(\boldsymbol{b}_{n+1}\right) \\
\text { and } \boldsymbol{\alpha}\left(\boldsymbol{a}_{n}\right)+\boldsymbol{\beta}\left(\boldsymbol{b}_{n+1}\right) \leq \psi\left(\boldsymbol{\alpha}\left(\boldsymbol{a}_{n-1}\right)\right) \boldsymbol{\alpha}\left(\boldsymbol{a}_{n-1}\right) \\
+\psi\left(\boldsymbol{\beta}\left(\boldsymbol{b}_{n}\right)\right) \boldsymbol{\beta}\left(\boldsymbol{b}_{n}\right) \\
<\boldsymbol{\alpha}\left(\boldsymbol{a}_{n-1}\right)+\boldsymbol{\beta}\left(\boldsymbol{b}_{n}\right)
\end{array}
$$

From (3) and (5) which shows that the bisequence $\left(\left\{\boldsymbol{\alpha}\left(\boldsymbol{a}_{\boldsymbol{n}}\right)\right\}\right.$, $\left.\left\{\boldsymbol{\beta}\left(\boldsymbol{b}_{\boldsymbol{n}}\right)\right\}\right)$ is non increasing bi-sequences of non-negative real numbers. So they must converges to $\lambda 1 ; \lambda 2 \geq 0$ respectively. Suppose $\lambda 1>0$ or $\lambda 2>0$. Letting $n \rightarrow \infty$ in equation (3) and (5), we get contradiction. Therefore,

$$
\lim _{n \rightarrow \infty} \alpha\left(a_{n}\right)=\lim _{n \rightarrow \infty} \beta\left(b_{n}\right)=0
$$

Now from (4), we have

$$
\sum_{n=1}^{m} \mathrm{~d}\left(\boldsymbol{a}_{n}, \boldsymbol{b}_{n+1}\right) \leq \mathrm{d}\left(\boldsymbol{a}_{1}, \boldsymbol{b}_{2}\right)+\mathrm{d}\left(\boldsymbol{a}_{2}, \boldsymbol{b}_{3}\right)+\cdots+\mathrm{d}\left(\boldsymbol{a}_{\boldsymbol{m}}, \boldsymbol{b}_{m+1}\right)
$$

$$
\begin{aligned}
& <\alpha\left(a_{0}\right)-\alpha\left(a_{1}\right)+\beta\left(b_{1}\right)-\beta\left(b_{2}\right)+\alpha\left(a_{1}\right)- \\
& \quad \alpha\left(a_{2}\right)+\beta\left(b_{2}\right)-\beta\left(b_{3}\right)+\cdots \cdots \cdots \\
& +\alpha\left(a_{m-1}\right)-\alpha\left(a_{m}\right)++\beta\left(b_{m}\right)-\beta\left(b_{m+1}\right) \\
& <\alpha\left(a_{0}\right)+\beta\left(b_{1}\right)
\end{aligned}
$$

This shows that $\sum_{n=1}^{m} \mathbf{d}\left(\boldsymbol{a}_{\boldsymbol{n}}, \boldsymbol{b}_{\boldsymbol{n}+\mathbf{1}}\right)$ is a bi-convergent series. Similarly, we can prove that $\sum_{n=1}^{m} \mathbf{d}\left(\boldsymbol{a}_{\boldsymbol{n}}, \boldsymbol{b}_{\boldsymbol{n}}\right)$ is a bi-convergent series. Hence convergent. Using the property (iv) in definition (1.1), for each n, $\mathrm{m} \in \boldsymbol{N}$ with $\mathrm{n}<\mathrm{m}$ and from (2) and (4). Then we have

$$
\begin{aligned}
& \mathbf{d}\left(a_{n}, b_{m}\right) \leq \mathrm{d}\left(a_{n}, b_{n+1}\right)+\mathrm{d}\left(a_{n+1}, b_{n+1}\right)+\cdots \\
& +\mathbf{d}\left(a_{m-1}, b_{m-1}\right)+\mathbf{d}\left(a_{m-1}, b_{m}\right) \\
& <\alpha\left(a_{n-1}\right)-\alpha\left(a_{n}\right)+\beta\left(b_{n}\right)-\beta\left(b_{n+1}\right)+\alpha\left(a_{n}\right)- \\
& \boldsymbol{\alpha}\left(\boldsymbol{a}_{n+1}\right)+\boldsymbol{\beta}\left(\boldsymbol{b}_{n+1}\right)-\boldsymbol{\beta}\left(\boldsymbol{b}_{n+2}\right)+\cdots \cdots \\
& +\alpha\left(a_{m-2}\right)-\alpha\left(a_{m-1}\right)++\beta\left(b_{m-1}\right)-\beta\left(b_{m}\right) \\
& \rightarrow \mathbf{0} \text { as } \mathrm{n}, \mathrm{m} \rightarrow \infty \text {. }
\end{aligned}
$$

Similarly, $\mathbf{d}\left(\boldsymbol{a}_{\boldsymbol{m}}, \boldsymbol{b}_{\boldsymbol{n}}\right) \rightarrow \mathbf{0}$ as $\mathrm{n}, \mathrm{m} \rightarrow \infty$. This shown that $\left(\boldsymbol{a}_{\boldsymbol{n}}, \boldsymbol{b}_{\boldsymbol{n}}\right)$ is Cauchy bi-sequence in $(\mathrm{U}, \mathrm{V})$. Therefore,

$\lim _{n \rightarrow \infty}\left(\boldsymbol{a}_{\boldsymbol{n}}, \boldsymbol{b}_{\boldsymbol{n}}\right)=\mathbf{0}$. Since $(\mathrm{U}, \mathrm{V}, \mathrm{d})$ is complete, $\left(\boldsymbol{a}_{\boldsymbol{n}}, \boldsymbol{b}_{\boldsymbol{n}}\right)$ is converges and thus bi-converges to point $\kappa \in \mathbf{U} \cap \mathbf{V}$ such that

$$
\lim _{n \rightarrow \infty} a_{n+1}=\kappa=\lim _{n \rightarrow \infty} b_{n+1}
$$

We prove that $\kappa$ is fixed point of $F$. Since $\boldsymbol{\alpha}, \boldsymbol{\beta}$ are lower semi continuous functions, so

$\lim _{n \rightarrow \infty} \alpha\left(a_{n}\right)=\alpha(\kappa), \lim _{n \rightarrow \infty} \beta\left(b_{n}\right)=\beta(\kappa)$ from (6), we get $\boldsymbol{\alpha}(\boldsymbol{\kappa})=\mathbf{0}=\boldsymbol{\beta}(\boldsymbol{\kappa})$. since $\lim _{\boldsymbol{n} \rightarrow \infty} \boldsymbol{F} \boldsymbol{a}_{\boldsymbol{n}+\mathbf{1}}=\boldsymbol{F} \boldsymbol{\kappa}$. From (1) and (iv) in definition (1.1), we have

$$
\begin{aligned}
\mathrm{d}(\mathrm{F} \kappa, \kappa) \leq \mathrm{d}\left(\mathrm{F} \kappa, \boldsymbol{b}_{n+2}\right)+\mathrm{d}\left(\boldsymbol{a}_{n+2}, \boldsymbol{b}_{n+2}\right)+\mathrm{d}\left(\boldsymbol{a}_{n+2}, \kappa\right) \\
\leq \mathrm{d}\left(\mathrm{F} \kappa, \boldsymbol{F} \boldsymbol{b}_{n+1}\right)+\mathrm{d}\left(\boldsymbol{a}_{n+2}, \boldsymbol{b}_{n+2}\right)+\mathrm{d}\left(\boldsymbol{a}_{n+2}, \kappa\right) \\
\leq \psi(\boldsymbol{\alpha}(\boldsymbol{\kappa})) \boldsymbol{\alpha}(\boldsymbol{\kappa})-\boldsymbol{\alpha}(\mathrm{F} \boldsymbol{\kappa}) \\
\quad+\psi\left(\boldsymbol{\beta}\left(\boldsymbol{b}_{n+1}\right)\right) \boldsymbol{\beta}\left(\boldsymbol{b}_{n+1}\right)-\boldsymbol{\beta}\left(\mathrm{F} \boldsymbol{b}_{n+1}\right) \\
\quad+\mathrm{d}\left(\boldsymbol{a}_{n+2}, \boldsymbol{b}_{n+2}\right)+\mathrm{d}\left(\boldsymbol{a}_{n+2}, \kappa\right) \\
\quad<(\boldsymbol{\alpha})-\boldsymbol{\alpha}(\mathrm{F} \boldsymbol{\kappa})+\boldsymbol{\beta}\left(\boldsymbol{b}_{n+1}\right)-\boldsymbol{\beta}\left(\boldsymbol{b}_{n+2}\right)+\mathrm{d}\left(\boldsymbol{a}_{n+2},\right. \\
\left.\boldsymbol{b}_{n+2}\right) \quad+\mathrm{d}\left(\boldsymbol{a}_{n+2}, \kappa\right) \rightarrow \mathbf{0} \text { as } \mathrm{n} \rightarrow \infty .
\end{aligned}
$$

Therefore, $\mathrm{d}(\mathrm{F} \kappa, \kappa)=0$ implies $\mathrm{F} \kappa=\kappa$. Now we prove the uniqueness, we begin by taking $v$ be another fixed point of covariant map F. Then $\mathrm{F} v=v$ implies $v \in U \cap V$ and we have

$\mathrm{d}(\kappa, v)=\mathrm{d}(\mathrm{F} \kappa, \mathrm{F} v) \leq \psi(\boldsymbol{\alpha}(\boldsymbol{\kappa})) \boldsymbol{\alpha}(\boldsymbol{\kappa})-\boldsymbol{\alpha}(\mathrm{F} \boldsymbol{\kappa})$

$$
+\psi(\boldsymbol{\beta}(\boldsymbol{v})) \boldsymbol{\beta}(\boldsymbol{v})-\boldsymbol{\beta}(\mathrm{F} \boldsymbol{v})
$$

$$
<\boldsymbol{\alpha}(\boldsymbol{\kappa})-\boldsymbol{\alpha}(\boldsymbol{\kappa})+\boldsymbol{\beta}(\boldsymbol{v})-\boldsymbol{\beta}(\boldsymbol{v})=0
$$

Therefore, $d(\kappa, v)=0$ implies $\kappa=v$. Hence $\kappa$ is unique fixed point of covariant mapping F.

Example 2.2: Let $U=\left\{U_{m}(R) / U_{m}(R)\right.$ is upper triangular matrices over R \} and 
$\mathrm{V}=\left\{\mathrm{L}_{\mathrm{m}}(\mathrm{R}) / \mathrm{L}_{\mathrm{m}}(\mathrm{R})\right.$ is lower triangular matrices over $\left.\mathrm{R}\right\}$. Define $\mathrm{d}: \mathrm{U}_{\mathrm{m}}(\mathrm{R}) \times \mathrm{L}_{\mathrm{m}}(\mathrm{R}) \rightarrow[0, \infty)$ by d $(\mathrm{P}, \mathrm{Q})=\sum_{\mathrm{I}, \mathrm{j}=1}^{\mathrm{m}}\left|\mathrm{p}_{\mathrm{ij}}-q_{\mathrm{ij}}\right|$ for all $\mathrm{P}=\left(\mathrm{p}_{\mathrm{ij}}\right)_{\mathrm{m} \times \mathrm{m}} \in \mathrm{U}_{\mathrm{m}}(\mathrm{R})$ and $\mathrm{Q}=\left(\mathrm{q}_{\mathrm{ij}}\right)_{\mathrm{m} \times \mathrm{m}} \in \mathrm{L}_{\mathrm{m}}(\mathrm{R})$. Then obviously, $(\mathrm{U}, \mathrm{V}, \mathrm{d})$ is a complete bipolar metric space.

Let $\mathrm{F}:(U, V) \rightrightarrows(\mathrm{U}, \mathrm{V})$ be defined as $\mathrm{F}(\mathrm{P})=\frac{1}{7}\left(p_{i j}\right)_{m \times m}$ for all $\mathrm{P}=\left(\mathrm{p}_{\mathrm{ij}}\right)_{\mathrm{m} \times \mathrm{m}}, \in U \cup V$. Let $\alpha, \beta: \mathrm{U} \cup V \rightarrow[0, \infty)$ be a lower semi continuous mappings be defined as $\alpha(P)=\sum_{i, j=1}^{m}\left|p_{i j}\right|$ and $\beta(P)=2 \sum_{i, j=1}^{m}\left|p_{i j}\right|$ for all $\mathrm{P}=\left(\mathrm{p}_{\mathrm{ij}}\right)_{\mathrm{m} \times \mathrm{m}^{\prime}} \in \mathrm{U}_{\mathrm{m}}(\mathrm{R}) \cup \mathrm{L}_{\mathrm{m}}(\mathrm{R})$. Define $\psi:(-\infty,+\infty) \rightarrow(0,1)$ as $\psi(\mathrm{t})= \begin{cases}\frac{3}{5}, & t>0 \\ 0, & t<0\end{cases}$

Now for each $\mathrm{P}, \mathrm{Q} \in U \cup V$, we have $\mathrm{d}(\mathrm{F} \mathrm{P},, \mathrm{F} \mathrm{Q})=\mathrm{d}\left(\frac{1}{7}\left(p_{i j}\right)_{m \times m} \frac{1}{7}\left(q_{i j}\right)_{m \times m}\right)$ $=\frac{1}{7} \sum_{\mathrm{i}, \mathrm{j}=1}^{\mathrm{m}}\left|\mathrm{p}_{\mathrm{ij}}-q_{\mathrm{ij}}\right| \leq \frac{16}{35} \sum_{\mathrm{i}, \mathrm{j}=1}^{\mathrm{m}}\left|\mathrm{p}_{\mathrm{ij}}-q_{\mathrm{ij}}\right|$ $\leq \frac{3}{5} \sum_{\mathrm{i}, \mathrm{j}=1}^{\mathrm{m}}\left|\mathrm{p}_{\mathrm{ij}}\right|-\frac{1}{7} \sum_{\mathrm{i}, \mathrm{j}=1}^{\mathrm{m}}\left|\mathrm{p}_{\mathrm{ij}}\right|+\frac{3}{5} \sum_{\mathrm{i}, \mathrm{j}=1}^{\mathrm{m}}\left|2 \mathrm{q}_{\mathrm{ij}}\right|-\frac{1}{7} \sum_{\mathrm{i}, \mathrm{j}=1}^{\mathrm{m}}\left|2 \mathrm{q}_{\mathrm{ij}}\right|$ $\leq \psi\left(\alpha\left(\left(p_{i j}\right)_{m \times m}\right)\right) \alpha\left(\left(p_{i j}\right)_{m \times m}\right)-\alpha\left(\frac{1}{7}\left(p_{i j}\right)_{m \times m}\right)$ $+\psi\left(\beta\left(\left(q_{i j}\right)_{m \times m}\right)\right) \beta\left(\left(q_{i j}\right)_{m \times m}\right)-\beta\left(\frac{1}{7}\left(q_{i j}\right)_{m \times m}\right)$

$\leq \psi(\alpha(P)) \alpha(P)-\alpha(F P)+\psi(\beta(Q)) \beta(Q)-\alpha(F Q)$

Thus, $F$ satisfy all the conditions of Theorem 2.1 and $\mathrm{O}_{\mathrm{m} \times \mathrm{m}}$, is unique fixed point of $\mathrm{F}$.

Theorem 2.3: Let $(\mathrm{U}, \mathrm{V}, \mathrm{d})$ be a complete bipolar metric spaces, suppose that $\mathrm{F}:(\mathrm{U}, \mathrm{V}) \rightrightarrows(\mathrm{U}, \mathrm{V})$ be a covariant mapping satisfies

$\mathrm{d}(\mathrm{Fa}, \mathrm{Fb}) \leq \boldsymbol{\alpha}(\boldsymbol{\psi}(\boldsymbol{a}, \boldsymbol{b})) \boldsymbol{\psi}(\mathrm{a}, \mathrm{b})-\psi(\mathrm{Fa}, \mathrm{Fb})$

(8) for all $\mathrm{a} \in \boldsymbol{U}, \mathrm{b} \in \boldsymbol{V}$, where $\psi: \mathrm{U} \times \mathbf{V} \rightarrow[0, \infty)$ is lower semi continuous function and $\boldsymbol{\alpha}:(-\infty, \infty) \rightarrow(0,1)$ be a continuous function and provided that $\mathrm{F}$ is continuous. Then the mapping $\quad \mathrm{F}: \mathrm{U} \cup \boldsymbol{V} \rightarrow$ $\boldsymbol{U} \cup \mathrm{V}$ has a unique fixed point.

Proof: Similarly to the above Theorem 2.1. We construct the bisequence $\left(\left\{\boldsymbol{a}_{\boldsymbol{n}}\right\},\left\{\boldsymbol{b}_{\boldsymbol{n}}\right\}\right)$ in (U, V) as F $\boldsymbol{a}_{\boldsymbol{n}}=\boldsymbol{a}_{\boldsymbol{n}+\mathbf{1}}$ and $\mathrm{F} \boldsymbol{b}_{\boldsymbol{n}}=\boldsymbol{b}_{\boldsymbol{n}+\boldsymbol{1}}$ for $\mathrm{n}=0,1,2, \cdots \cdots$

Now from (8), we have

$$
\begin{aligned}
& \mathrm{d}\left(\boldsymbol{a}_{n}, \boldsymbol{b}_{n+1}\right)=\mathrm{d}\left(\mathrm{F} a_{n-1}, \mathrm{~F} b_{n}\right) \\
& \leq \alpha\left(\psi\left(a_{n-1}, b_{n}\right)\right) \psi\left(a_{n-1}, b_{n}\right)-\psi\left(F a_{n-1}, F b_{n}\right) \\
& \leq \alpha\left(\psi\left(a_{n-1}, b_{n}\right)\right) \psi\left(a_{n-1}, b_{n}\right)-\psi\left(a_{n}, b_{n+1}\right) \\
& <\psi\left(a_{n-1}, b_{n}\right)-\psi\left(a_{n}, b_{n+1}\right) \\
& \psi\left(a_{n}, b_{n+1}\right)<\psi\left(a_{n-1}, b_{n}\right) \alpha\left(\psi\left(a_{n-1}, b_{n}\right)\right) \\
& <\psi\left(a_{n-1}, b_{n}\right)
\end{aligned}
$$

Also,

$$
\begin{aligned}
& \mathrm{d}\left(\boldsymbol{a}_{n}, \boldsymbol{b}_{n}\right)=\mathrm{d}\left(\mathrm{F} a_{n-1}, \mathrm{~F} b_{n-1}\right) \\
& \leq \alpha\left(\psi\left(a_{n-1}, b_{n-1}\right)\right) \psi\left(a_{n-1}, b_{n-1}\right)-\psi\left(F a_{n-1}, F b_{n-1}\right) \\
& \leq \alpha\left(\psi\left(a_{n-1}, b_{n-1}\right)\right) \psi\left(a_{n-1}, b_{n-1}\right)-\psi\left(a_{n}, b_{n}\right) \\
& <\psi\left(a_{n-1}, b_{n-1}\right)-\psi\left(a_{n}, b_{n}\right) \\
& \text { And } \psi\left(a_{n}, b_{n}\right)<\psi\left(a_{n-1}, b_{n-1}\right) \alpha\left(\psi\left(a_{n-1}, b_{n-1}\right)\right) \\
& <\psi\left(a_{n-1}, b_{n-1}\right)
\end{aligned}
$$

Equations (10) and (12) shows that the bisequence

$\left(\left\{\boldsymbol{\psi}\left(\boldsymbol{a}_{\boldsymbol{n}}, \boldsymbol{b}_{\boldsymbol{n}}\right)\right\},\right)$ is non increasing bi-sequences of non-negative real numbers. So they must converges to $\lambda 1 \geq 0$. Suppose $\lambda 1>0$. Letting $n \rightarrow \infty$ in equation (10) and (12), we get contradiction. Therefore,

$$
\lim _{n \rightarrow \infty} \psi\left(a_{n}, b_{n}\right)=0
$$

Now, from (9), we have

$$
\begin{aligned}
\sum_{n=1}^{m} \mathrm{~d}\left(\boldsymbol{a}_{n}, \boldsymbol{b}_{n+1}\right) \leq \mathrm{d}\left(\boldsymbol{a}_{1}, \boldsymbol{b}_{2}\right)+\mathrm{d}\left(\boldsymbol{a}_{2}, \boldsymbol{b}_{3}\right)+\cdots+\mathrm{d}\left(\boldsymbol{a}_{m}, \boldsymbol{b}_{m+1}\right) \\
<\boldsymbol{\psi}\left(\boldsymbol{a}_{0,}, \boldsymbol{b}_{1}\right)-\left(\boldsymbol{a}_{1}, \boldsymbol{b}_{2}\right)+\boldsymbol{\psi}\left(\boldsymbol{a}_{1}, \boldsymbol{b}_{2}\right)+\cdots \cdots \cdots \\
+\boldsymbol{\psi}\left(\boldsymbol{a}_{\boldsymbol{m}-1}, \boldsymbol{b}_{\boldsymbol{m}}\right)-\boldsymbol{\psi}\left(\boldsymbol{a}_{\boldsymbol{m}}, \boldsymbol{b}_{m+1}\right) \\
<\boldsymbol{\psi}\left(\boldsymbol{a}_{0}, \boldsymbol{b}_{1}\right) \text { This shows that }
\end{aligned}
$$

$\sum_{n=\mathbf{1}}^{\boldsymbol{m}} \mathbf{d}\left(\boldsymbol{a}_{\boldsymbol{n}}, \boldsymbol{b}_{\boldsymbol{n}+\mathbf{1}}\right)$ is a bi-convergent series. Similarly, we can prove that $\sum_{n=1}^{m} d\left(a_{n}, b_{n}\right)$ is a biconvergent series. Hence convergent. Using the property (iv) in definition

(1.1), for each $\mathrm{n}, \mathrm{m} \in \mathbf{N}$ with $\mathrm{n}<\mathrm{m}$, from (9) and (11). Then we have

$$
\begin{aligned}
\mathbf{d}\left(\boldsymbol{a}_{n}, \boldsymbol{b}_{m}\right) \leq \mathbf{d}\left(\boldsymbol{a}_{n}, \boldsymbol{b}_{n+1}\right)+\mathbf{d}\left(\boldsymbol{a}_{n+1}, \boldsymbol{b}_{n+1}\right)+\cdots \\
\quad+\mathbf{d}\left(\boldsymbol{a}_{m-1}, \boldsymbol{b}_{m-1}\right)+\mathbf{d}\left(\boldsymbol{a}_{m-1}, \boldsymbol{b}_{m}\right) \\
<\boldsymbol{\psi}\left(\boldsymbol{a}_{n-1}, \boldsymbol{b}_{n}\right)-\boldsymbol{\psi}\left(\boldsymbol{a}_{n}, \boldsymbol{b}_{n+1}\right) \\
+\psi\left(\boldsymbol{a}_{n}, \boldsymbol{b}_{n+1}\right)-\boldsymbol{\psi}\left(\boldsymbol{a}_{n+1}, \boldsymbol{b}_{n+2}\right)+\cdots \cdots \\
\quad+\boldsymbol{\psi}\left(\boldsymbol{a}_{m-2}, \boldsymbol{b}_{m-1}\right)-\boldsymbol{\psi}\left(\boldsymbol{a}_{m-1}, \boldsymbol{b}_{m}\right) \\
\rightarrow \mathbf{0} \text { as } \mathrm{n}, \mathrm{m} \rightarrow \infty .
\end{aligned}
$$

Similarly, $\mathbf{d}\left(\boldsymbol{a}_{\boldsymbol{m}}, \boldsymbol{b}_{\boldsymbol{n}}\right) \rightarrow \mathbf{0}$ as $\mathrm{n}, \mathrm{m} \rightarrow \infty$. This shown that $\left(\mathbf{a}_{\mathbf{n}}, \mathbf{b}_{\mathbf{n}}\right)$ is Cauchy bi-sequence in $(\mathrm{U}, \mathrm{V})$. Therefore,

$\lim _{n \rightarrow \infty}\left(\boldsymbol{a}_{\boldsymbol{n}}, \boldsymbol{b}_{\boldsymbol{n}}\right)=\mathbf{0}$. Since $(\mathrm{U}, \mathrm{V}, \mathrm{d})$ is complete, $\left(\boldsymbol{a}_{\boldsymbol{n}}, \boldsymbol{b}_{\boldsymbol{n}}\right)$ is converges and thus bi-converges to point $\kappa \in \mathbf{U} \cap \mathbf{V}$ such that

$$
\lim _{n \rightarrow \infty} a_{n+1}=\kappa=\lim _{n \rightarrow \infty} b_{n+1}
$$

We prove that $\kappa$ is fixed point of $F$. Since $F$ is continuous function, So $\lim _{\boldsymbol{n} \rightarrow \infty} \boldsymbol{F} \boldsymbol{a}_{\boldsymbol{n}+\mathbf{1}}=\boldsymbol{F} \boldsymbol{\kappa}$. Since $\psi$ is lower semi continuous function and

$\lim _{n \rightarrow \infty} \psi\left(a_{n}, b_{n}\right)=\psi(\kappa, \kappa)$. From (13), we get

$\psi(\kappa, \kappa) .=0$. From (8) and (iv) in definition (1.1), we have

$$
\begin{array}{r}
\mathrm{d}(\mathrm{F} \kappa, \kappa) \leq \mathrm{d}\left(\mathrm{F} \kappa, \boldsymbol{b}_{n+2}\right)+\mathrm{d}\left(\boldsymbol{a}_{n+2}, \boldsymbol{b}_{n+2}\right)+\mathrm{d}\left(\boldsymbol{a}_{n+2}, \kappa\right) \\
\leq \mathrm{d}\left(\mathrm{F} \kappa, \boldsymbol{F b}_{n+1}\right)+\mathrm{d}\left(\boldsymbol{a}_{n+2}, \boldsymbol{b}_{n+2}\right)+\mathrm{d}\left(\boldsymbol{a}_{n+2}, \kappa\right) \\
\leq \boldsymbol{\alpha}\left(\boldsymbol{\psi}\left(\boldsymbol{\kappa}, \boldsymbol{b}_{n+2}\right)\right) \boldsymbol{\psi}\left(\boldsymbol{\kappa}, \boldsymbol{b}_{n+2}\right)-\boldsymbol{\psi}\left(\boldsymbol{F} \boldsymbol{\kappa}, \boldsymbol{F} \boldsymbol{b}_{n+2}\right) \\
\quad+\mathrm{d}\left(\boldsymbol{a}_{n+2}, \boldsymbol{b}_{n+2}\right)+\mathrm{d}\left(\boldsymbol{a}_{n+2}, \kappa\right) \rightarrow \mathbf{0} \text { as } \mathrm{n} \rightarrow \infty .
\end{array}
$$

Therefore, $\mathrm{d}(\mathrm{F} \kappa, \kappa)=0$ implies $\mathrm{F} \kappa=\kappa$.

Uniqueness follows from the Theorem 2.1.

\section{Applications}

\subsection{Application to the Existence of Solutions of Integral Equations}

Theorem 3.1: Let us consider the integral equation

$\boldsymbol{\gamma}(\kappa)=\mathrm{f}(\kappa)+\int \boldsymbol{S}(\boldsymbol{\kappa}, \boldsymbol{v}, \boldsymbol{\gamma}(\boldsymbol{v})) \mathrm{d} v, \kappa \in \boldsymbol{E}_{\mathbf{1}} \cup \boldsymbol{E}_{2}$

$\mathbf{E}_{1} \cup \mathbf{E}_{\mathbf{2}}$ is Lebesgue measurable set with where $\left.\mathbf{E}_{2}\right)<\infty$. Suppose that

(i) S: $\left(\boldsymbol{E}_{\mathbf{1}}{ }^{2} \cup \boldsymbol{E}_{2}{ }^{2}\right) \times[\mathbf{0},+\infty) \rightarrow[0,+\infty)$ and $\mathrm{m}\left(\mathbf{E}_{\mathbf{1}} \mathrm{U}\right.$

$\boldsymbol{L}^{\infty}\left(\boldsymbol{E}_{\mathbf{1}}\right) \cup \boldsymbol{L}^{\infty}\left(\boldsymbol{E}_{\mathbf{2}}\right)$

(ii) There is a continuous function $\Gamma: E_{1}{ }^{2} \cup E_{2}{ }^{2} \rightarrow[0, \infty)$ such that for all $(\kappa, v) \in{E_{1}}^{2} \cup{E_{2}}^{2}$ $|S(\kappa, v, \gamma(v))-S(\kappa, v, \beta(v))| \leq \frac{1}{3} \Gamma(\kappa, v)|\gamma(v)-\beta(v)|$.

(iii) $\quad\left\|\int \Gamma(\kappa, v) \mathrm{d} v\right\| \leq 1$ i.e $\sup _{\kappa \in E_{1} \cup E_{2}} \int|\Gamma(\kappa, v) \mathrm{d} v|$ $\leq 1$.

Then The equation has unique solution in $L^{\infty}\left(E_{1}\right) \cup L^{\infty}\left(E_{2}\right)$ 
Proof: Let $\mathrm{U}=\boldsymbol{L}^{\infty}\left(\boldsymbol{E}_{\mathbf{1}}\right)$ and $\mathrm{V}=\boldsymbol{L}^{\infty}\left(\boldsymbol{E}_{2}\right)$ be two normed linear spaces, where $\boldsymbol{E}_{\mathbf{1}}, \boldsymbol{E}_{\mathbf{2}}$ are two Lebesgue measurable sets with $\mathrm{m}\left(\boldsymbol{E}_{\mathbf{1}} \cup \boldsymbol{E}_{\mathbf{2}}\right)<\infty$. Consider d: $\mathrm{U} \times \mathrm{V} \rightarrow[\mathbf{0}, \infty)$ be defined by $\mathrm{d}(\mathrm{f}, \mathrm{g})=\|\boldsymbol{f}-\boldsymbol{g}\|_{\infty}$ for all $(\mathrm{f}, \mathrm{g}) \in \mathrm{U} \times \mathrm{V}$. Then $(\mathrm{U}, \mathrm{V}, \mathrm{d})$ is complete bipolar metric spaces. Define covariant map F: $\boldsymbol{U} \cup \boldsymbol{V} \rightarrow \boldsymbol{U} \cup \boldsymbol{V}$ by $\mathrm{F}(\boldsymbol{\gamma}(\kappa))=\int \boldsymbol{S}(\boldsymbol{\kappa}, \boldsymbol{v}, \boldsymbol{\gamma}(\boldsymbol{v})) \mathrm{d} v+\mathrm{f}(\kappa) \kappa \in \boldsymbol{E}_{\mathbf{1}} \cup \boldsymbol{E}_{\mathbf{2}}$. Define $\psi: \mathrm{U} \times \mathrm{V} \rightarrow[0, \infty)$ by $\psi(\boldsymbol{\gamma}(\boldsymbol{v}), \boldsymbol{\beta}(\boldsymbol{v}))=3\|\boldsymbol{\gamma}(\boldsymbol{v})-\boldsymbol{\beta}(\boldsymbol{v})\|$ and define $\alpha:(-\infty,+\infty) \rightarrow(0,1)$ by $\alpha(\mathrm{t})=\left\{\begin{aligned} \frac{7}{12}, & t>0 \\ 0, & t<0\end{aligned}\right.$

Notice that $\mathrm{d}(\mathrm{F} \boldsymbol{\gamma}(\boldsymbol{v}), \boldsymbol{F} \boldsymbol{\beta}(\boldsymbol{v}))=\|\mathbf{F} \boldsymbol{\gamma}(\boldsymbol{v})-\boldsymbol{F} \boldsymbol{\beta}(\boldsymbol{v})\|$

$=\left\|\int S(\kappa, v, \gamma(v)) \mathrm{d} v+\mathbf{f}(\kappa)-\int S(\kappa, v, \beta(v)) \mathrm{d} v-\mathbf{f}(\kappa)\right\|$

$=\left|\int S(\kappa, v, \gamma(v)) \mathrm{d} v-\int S(\kappa, v, \beta(v)) \mathrm{d} v\right|$

$\leq \int|S(\kappa, v, \gamma(v))-S(\kappa, v, \beta(v))| \mathrm{d} v$

$\leq \frac{1}{3} \int \Gamma(\kappa, v)|\gamma(v)-\beta(v)| d v$

$\leq \frac{1}{3}\|\gamma(v)-\beta(v)\|_{\infty} \int\|\Gamma(\kappa, v)\| \mathrm{d} v$

Then $\mathrm{d}(\mathrm{F}$

$F \beta(v)) \leq\|\gamma(v)-\beta(v)\| \sup _{\kappa \in E_{1} \cup E_{2}} \int|\Gamma(\kappa, v) \mathrm{d} v|$.

$$
\begin{aligned}
& \quad \leq \frac{1}{3}\|\gamma(\boldsymbol{v})-\beta(v)\|_{\infty} \leq \frac{3}{4}\|\gamma(\boldsymbol{v})-\beta(v)\|_{\infty} \\
& =\frac{7}{12} \times 3\|\gamma(\boldsymbol{v})-\beta(v)\|_{\infty}-3\|\boldsymbol{F} \gamma(\boldsymbol{v})-\boldsymbol{F} \beta(\boldsymbol{v})\| \\
& \leq \boldsymbol{\alpha}(\boldsymbol{\psi}(\boldsymbol{\gamma}, \boldsymbol{\beta})) \psi(\boldsymbol{\gamma}, \boldsymbol{\beta})-\psi(\boldsymbol{F} \gamma, \boldsymbol{F} \boldsymbol{\beta})
\end{aligned}
$$

Thus F satisfy all the conditions of Theorem 2.3. Then F has a unique fixed point in UUV.

\subsection{Application to Homotopy Theory}

Theorem 3.2: Let $(\mathrm{U}, \mathrm{V}, \mathrm{d})$ be a complete bipolar metric spaces, (A, B) be an open subset of $(\mathrm{U}, \mathrm{V})$ and $(\bar{A}, \bar{B})$ be a closed subset of $(\mathrm{U}, \mathrm{V})$ such that $(\mathrm{A}, \mathrm{B}) \subseteq(\bar{A}, \bar{B})$. Suppose $\mathrm{H}:(\bar{A}, \cup \bar{B}) \times[0$, 1] $\rightarrow \mathrm{UUV}$ be an operator with following conditions are satisfying. $\mathrm{x} \neq \mathrm{H}(\mathrm{x}, \kappa)$ for each $\mathrm{x} \in \partial \mathrm{A} \cup \partial \mathrm{B}$ and $\kappa \in[0,1]$ (Here $\partial \mathrm{A} \cup \partial \mathrm{B}$ is boundary of $\mathrm{A} \cup \mathrm{B}$ in $\mathrm{U} \cup \mathrm{V}$ ) $\mathrm{d}(H(x, \kappa), H(y, \kappa)) \leq \psi(\alpha(x)) \alpha(x)-\alpha(H(x, \kappa))$

$$
+\psi(\beta(y)) \beta(y)-\beta(H(y, \kappa))
$$
for all $\mathrm{x} \in \bar{A}, \quad \mathrm{y} \in \bar{B}$ and $\kappa \in[0,1]$, where $\alpha, \beta: \mathrm{U} \cup \mathrm{V} \rightarrow[0, \infty)$ are lower semi continuous functions and $\psi:(-\infty,+\infty) \rightarrow(0,1)$ is continuous function

(iii) $\quad \exists \mathrm{M} \geq 0 \ni \mathrm{d}(\mathrm{H}(\mathrm{x}, \kappa), \mathrm{H}(\mathrm{y}, \xi)) \leq \mathrm{M} \mid \kappa-\xi$ for every $\mathrm{x} \in \bar{A}$ and $\mathrm{y} \in \bar{B}$ and $\kappa, \xi \in[0,1]$.

Then $\mathrm{H}(., 0)$ has a fixed point $\Leftrightarrow \mathrm{H}(., 1)$ has a fixed point.

Proof. Let the set

$$
X=\{\kappa \in[0,1]: x=H(x, \kappa) \text { for some } x \in A\} .
$$$$
\mathrm{Y}=\{\xi \in[0,1]: \mathrm{y}=\mathrm{H}(\mathrm{y}, \xi) \text { for some } \mathrm{y} \in \mathrm{B}\} \text {. }
$$

Since $\mathrm{H}(; 0)$ has fixed point in $\mathrm{A} \cup \mathrm{B}$. So $0 \in \mathrm{X} \cap \mathrm{Y}$.That is $\mathrm{X \cap Y}$ is non-empty.

Now we show that $\mathrm{X} \cap \mathrm{Y}$ is both closed and open in $[0,1]$. Hence by the connectedness $\mathrm{X}=\mathrm{Y}=[0,1]$.

Let $\left(\left\{\kappa_{n}\right\},\left\{\xi_{n}\right\}\right) \subseteq(X, Y)$ with $\left(\kappa_{n}, \xi_{n}\right) \rightarrow(\kappa, \xi) \in[0,1]$ as $n \rightarrow \infty$. We must show that $\kappa=\xi \in X \cap Y$.

Since $\left(\kappa_{n}, \xi_{n}\right) \in(\mathrm{X}, \mathrm{Y})$ for $\mathrm{n}=0,1,2 \cdots \cdots$, There exists bisequence $\left(x_{n}, y_{n}\right)$ with $x_{n+1}=\mathrm{H}\left(x_{n}, \kappa_{n}\right)$ and $y_{n+1}=\mathrm{H}\left(y_{n}, \xi_{n}\right)$

Consider,

$$
\begin{aligned}
\mathrm{d}\left(x_{n}, y_{n+1}\right) & =\mathrm{d}\left(H\left(x_{n-1}, \kappa_{n-1}\right), H\left(y_{n}, \xi_{n}\right)\right) \\
& \leq \psi\left(\alpha\left(x_{n-1}\right)\right) \alpha\left(x_{n-1}\right)-\alpha\left(H\left(x_{n-1}, \kappa_{n-1}\right)\right) \\
& +\psi\left(\beta\left(y_{n}\right)\right) \beta\left(y_{n}\right)-\beta\left(H\left(y_{n}, \xi_{n}\right)\right) \\
< & \alpha\left(x_{n-1}\right)-\alpha\left(x_{n}\right)+\beta\left(y_{n}\right)-\beta\left(y_{n+1}\right) .
\end{aligned}
$$

$$
\begin{gathered}
\alpha\left(x_{n}\right)+\beta\left(y_{n+1}\right) \leq \psi\left(\alpha\left(x_{n-1}\right)\right) \alpha\left(x_{n-1}\right)-\alpha\left(x_{n-1}\right) \\
+\psi\left(\beta\left(y_{n}\right)\right) \beta\left(y_{n}\right)-\beta\left(y_{n}\right) \\
<\alpha\left(x_{n-1}\right)+\beta\left(y_{n}\right)
\end{gathered}
$$

Also we have

$$
\begin{aligned}
\mathrm{d}\left(x_{n}, y_{n}\right)= & \mathrm{d}\left(H\left(x_{n-1}, \kappa_{n-1}\right), H\left(y_{n-1}, \xi_{n-1}\right)\right) \\
& \leq \psi\left(\alpha\left(x_{n-1}\right)\right) \alpha\left(x_{n-1}\right)-\alpha\left(H\left(x_{n-1}, \kappa_{n-1}\right)\right) \\
& \quad+\psi\left(\beta\left(y_{n-1}\right)\right) \beta\left(y_{n-1}\right)-\beta\left(H\left(y_{n-1}, \xi_{n-1}\right)\right) \\
< & \alpha\left(x_{n-1}\right)-\alpha\left(x_{n}\right)+\beta\left(y_{n-1}\right)-\beta\left(y_{n}\right) .
\end{aligned}
$$

And

$$
\begin{aligned}
\alpha\left(x_{n}\right)+\beta\left(y_{n}\right) \leq & \psi\left(\alpha\left(x_{n-1}\right)\right) \alpha\left(x_{n-1}\right)-\alpha\left(x_{n-1}\right) \\
& +\psi\left(\beta\left(y_{n-1}\right)\right) \beta\left(y_{n-1}\right)-\beta\left(y_{n-1}\right) \\
< & \alpha\left(x_{n-1}\right)+\beta\left(y_{n-1}\right)
\end{aligned}
$$

From (16) and (18) which shows the bisequence

$\left(\left\{\alpha\left(x_{n}\right)\right\},\left\{\beta\left(y_{n}\right)\right\}\right)$ is non-increasing bisequence of nonnegative real numbers. So they must converges to $\lambda_{1} \geq 0$. Suppose $\lambda_{1}>0$ and letting $n \rightarrow \infty$ in equations (16) and (18), we get contradiction. Therefore

$$
\lim _{n \rightarrow \infty} \alpha\left(x_{n}\right)=\lim _{n \rightarrow \infty} \beta\left(y_{n}\right)=\mathbf{0}
$$

Now from (15), we have

$$
\begin{aligned}
\sum_{n=1}^{m} \mathrm{~d}\left(x_{n}, y_{n+1}\right) \leq & \mathrm{d}\left(x_{1}, y_{2}\right)+\mathrm{d}\left(x_{2}, y_{3}\right)+\cdots+\mathrm{d}\left(x_{m}, y_{m+1}\right) \\
< & \alpha\left(x_{0}\right)-\alpha\left(x_{1}\right)+\beta\left(y_{1}\right)-\beta\left(y_{2}\right)+\alpha\left(x_{1}\right)- \\
& \alpha\left(x_{2}\right)+\beta\left(y_{2}\right)-\beta\left(y_{3}\right)+\cdots \cdots \cdots \\
& +\alpha\left(x_{m-1}\right)-\alpha\left(x_{m}\right)++\beta\left(y_{m}\right)-\beta\left(y_{m+1}\right) \\
< & \alpha\left(x_{0}\right)+\beta\left(y_{1}\right)
\end{aligned}
$$

This shows that $\sum_{n=1}^{m} \mathbf{d}\left(\boldsymbol{x}_{\boldsymbol{n}}, \boldsymbol{y}_{\boldsymbol{n}+\mathbf{1}}\right)$ is a bi-convergent series. Similarly, we can prove that $\sum_{n=1}^{m} \mathbf{d}\left(\boldsymbol{x}_{n}, \boldsymbol{y}_{\boldsymbol{n}}\right)$ is a bi-convergent series. Hence convergent. Using the property (iv) in definition (1.1), for each $n, m \in \mathbf{N}$ with $n<m$. Then we have

$$
\begin{aligned}
& \mathrm{d}\left(x_{n}, y_{m}\right) \leq \mathrm{d}\left(x_{n}, y_{n+1}\right)+\mathrm{d}\left(x_{n+1}, y_{n+1}\right)+\cdots \\
& \leq d \\
& +\mathrm{d}\left(x_{m-1}, y_{m-1}\right)+\mathrm{d}\left(x_{m-1}, y_{m}\right) \\
& \left(\boldsymbol{H}\left(\boldsymbol{x}_{\boldsymbol{n}-1}, \boldsymbol{\kappa}_{n-1}\right), \boldsymbol{H}\left(\boldsymbol{y}_{n}, \boldsymbol{\xi}_{\boldsymbol{n}}\right)\right)+ \\
& \boldsymbol{d}\left(\boldsymbol{H}\left(\boldsymbol{x}_{n}, \boldsymbol{\kappa}_{n}\right), \boldsymbol{H}\left(\boldsymbol{y}_{n}, \xi_{n}\right)\right)+\cdots \cdots+d \\
& +\left(H\left(x_{m-2}, \kappa_{m-2}\right), H\left(y_{m-1}, \xi_{m-1}\right)\right) \\
& <\alpha\left(x_{n-1}\right)-\alpha\left(x_{n}\right)+\beta\left(y_{n}\right)-\beta\left(y_{n+1}\right)+\mathrm{M}\left|\kappa_{n}-\xi_{n}\right| \\
& +\mathrm{M}\left|\kappa_{m-2}-\xi_{m-2}\right| \\
& +\alpha\left(x_{m-2}\right)-\alpha\left(x_{m-1}\right)++\beta\left(y_{m-1}\right)-\beta\left(y_{m}\right) \\
& \rightarrow \mathbf{0} \text { as } \mathrm{n}, \mathrm{m} \rightarrow \infty \text {. }
\end{aligned}
$$

Similarly, $\mathbf{d}\left(\boldsymbol{x}_{\boldsymbol{m}}, \boldsymbol{y}_{\boldsymbol{n}}\right) \rightarrow \mathbf{0}$ as $\mathrm{n}, \mathrm{m} \rightarrow \infty$. This shown that $\left(\boldsymbol{x}_{\boldsymbol{n}}, \boldsymbol{y}_{\boldsymbol{m}}\right)$ is Cauchy bi-sequence in (A, B). By the completeness, there exist $\boldsymbol{\eta} \in \boldsymbol{A}$ and $\mu \in \boldsymbol{B}$ such that

$$
\lim _{n \rightarrow \infty} x_{n}=\mu \text { and } \quad \lim _{n \rightarrow \infty} y_{n}=\eta
$$

Now consider,

$\mathrm{d}(\mathrm{H}(\boldsymbol{\eta}, \kappa), \mu) \leq \mathrm{d}\left(\mathrm{H}(\boldsymbol{\eta}, \kappa), \boldsymbol{y}_{\boldsymbol{n}+\mathbf{1}}\right)+\mathrm{d}\left(\boldsymbol{x}_{\boldsymbol{n}+\mathbf{1}}, \boldsymbol{y}_{\boldsymbol{n}+\mathbf{1}}\right)+\mathrm{d}\left(\boldsymbol{x}_{\boldsymbol{n}+\mathbf{1}}, \mu\right)$

$$
\begin{aligned}
& \leq \mathrm{d}\left(\mathrm{H}(\boldsymbol{\eta}, \kappa), \boldsymbol{H}\left(\boldsymbol{y}_{\boldsymbol{n}}, \boldsymbol{\xi}_{\boldsymbol{n}}\right)\right)+\mathrm{d}\left(\mathrm{H}\left(\boldsymbol{x}_{\boldsymbol{n}}, \boldsymbol{\kappa}_{\boldsymbol{n}}\right), \mathrm{H}\left(\boldsymbol{y}_{\boldsymbol{n}}, \boldsymbol{\xi}_{\boldsymbol{n}}\right)\right) \\
&+\mathrm{d}\left(\boldsymbol{x}_{\boldsymbol{n}+\boldsymbol{1}}, \mu\right) \\
& \leq \boldsymbol{\psi}(\boldsymbol{\alpha}(\boldsymbol{\eta})) \boldsymbol{\alpha}(\boldsymbol{\eta})-\boldsymbol{\alpha}(\mathbf{H}(\boldsymbol{\eta}, \boldsymbol{\kappa}))+\boldsymbol{\psi}\left(\boldsymbol{\beta}\left(\boldsymbol{y}_{\boldsymbol{n}}\right)\right) \boldsymbol{\beta}\left(\boldsymbol{y}_{\boldsymbol{n}}\right)
\end{aligned}
$$




$$
\begin{array}{r}
-\boldsymbol{\beta}\left(\boldsymbol{H}\left(\boldsymbol{y}_{\boldsymbol{n}}, \xi_{\boldsymbol{n}}\right)\right)+\mathrm{M}\left|\boldsymbol{\kappa}_{\boldsymbol{n}}-\xi_{\boldsymbol{n}}\right|+\mathrm{d}\left(\boldsymbol{x}_{\boldsymbol{n}+\mathbf{1}}, \mu\right) \\
<\boldsymbol{\alpha}(\boldsymbol{\eta})-\boldsymbol{\alpha}(\mathbf{H}(\boldsymbol{\eta}, \boldsymbol{\kappa}))+\boldsymbol{\beta}\left(\boldsymbol{y}_{\boldsymbol{n}}\right)+\boldsymbol{\beta}\left(\boldsymbol{y}_{\boldsymbol{n}+\mathbf{1}}\right)+ \\
+\mathrm{M}\left|\boldsymbol{\kappa}_{\boldsymbol{n}}-\boldsymbol{\xi}_{\boldsymbol{n}}\right|+\mathrm{d}\left(\boldsymbol{x}_{\boldsymbol{n}+\mathbf{1}}, \mu\right) \rightarrow 0 \text { as } \mathrm{n} \rightarrow \infty .
\end{array}
$$

It follows that $\mathrm{d}(\mathrm{H}(\boldsymbol{\eta}, \kappa), \mu)=\mathbf{0}$ implies $\mathrm{H}(\boldsymbol{\eta}, \kappa)=\mu$. Similarly, we obtain $\mathrm{H}(\mu, \xi)=\boldsymbol{\eta}$. On the other hand, from (20), we have

$\mathrm{d}(\boldsymbol{\eta}, \mu)=\mathrm{d}\left(\lim _{n \rightarrow \infty} \boldsymbol{y}_{\boldsymbol{n}}, \lim _{n \rightarrow \infty} \boldsymbol{x}_{\boldsymbol{n}}\right)=\lim _{n \rightarrow \infty} d\left(x_{\boldsymbol{n}}, \boldsymbol{y}_{\boldsymbol{n}}\right)=0$.

Thus, $\boldsymbol{\eta}=\mu$. Therefore, $\kappa=\xi \in \mathrm{X} \cap \mathrm{Y}$. Clearly, $\mathrm{X} \cap \mathrm{Y}$ is closed in $[0,1]$

Since $\left(\kappa_{0}, \xi_{0}\right) \in(\mathrm{X}, \mathrm{Y})$, then there exists bisequence $\left(x_{0}, y_{0}\right)$

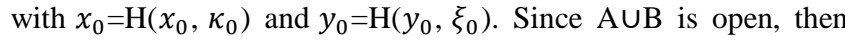
there exist $\mathrm{r}>0$ such that $\mathcal{B}_{d}\left(x_{0}, \mathrm{r}\right) \subseteq \mathrm{A} \cup \mathrm{B}$ and $\mathcal{B}_{d}\left(r, y_{0}\right) \subseteq \mathrm{A} \cup \mathrm{B}$. Choose $\kappa \in\left(\xi_{0}-\epsilon, \xi_{0}+\epsilon\right)$ such that $\left|\kappa-\xi_{0}\right| \leq \frac{1}{M^{n}}<\frac{\epsilon}{3}$ and

$\xi \in\left(\kappa_{0}-\epsilon, \kappa_{0}+\epsilon\right)$ such that $\left|\xi-\kappa_{0}\right| \leq \frac{1}{M^{n}}<\frac{\epsilon}{3}$ also $\left|\kappa_{0}-\xi_{0}\right| \leq \frac{1}{M^{n}}<\frac{\epsilon}{3}$. Then $\mathrm{y} \in \overline{\mathcal{B}_{X \cup Y}}\left(x_{0}, \mathrm{r}\right)=\left\{\mathrm{y}, y_{0} \in \mathrm{B} / \mathrm{d}\left(x_{0}, \mathrm{y}\right) \leq \mathrm{r}+\mathrm{d}\left(x_{0}, y_{0}\right)\right\}$ and $\mathrm{x} \in \overline{\mathcal{B}_{X \cup Y}}\left(r, y_{0}\right)=\left\{\mathrm{x}, x_{0} \in \mathrm{A} / \mathrm{d}\left(x, y_{0}\right) \leq \mathrm{r}+\mathrm{d}\left(x_{0}, y_{0}\right)\right\}$, also $\mathrm{d}\left(\mathrm{H}(\mathrm{x}, \kappa), y_{0}\right)=\mathrm{d}\left(\mathrm{H}(\mathrm{x}, \kappa), H\left(y_{0}, \xi_{0}\right)\right)$

$$
\begin{gathered}
\leq \mathrm{d}\left(\mathrm{H}(\mathrm{x}, \kappa), H\left(y, \xi_{0}\right)\right)+\mathrm{d}\left(\mathrm{H}\left(x_{0}, \kappa\right), H\left(y, \xi_{0}\right)\right) \\
\quad+\mathrm{d}\left(\mathrm{H}\left(x_{0}, \kappa\right), H\left(y_{0}, \xi_{0}\right)\right) \\
\leq 2 \mathrm{M}\left|\kappa-\xi_{0}\right|+\psi\left(\alpha\left(x_{0}\right)\right) \alpha\left(x_{0}\right)-\alpha\left(\mathrm{H}\left(x_{0}, \kappa\right)\right) \\
\left.+\psi(\beta(y)) \beta(y)-\beta\left(H\left(y, \xi_{0}\right)\right)\right) \\
\left.<\frac{2}{M^{n-1}}+\alpha\left(x_{0}\right)-\alpha\left(\mathrm{H}\left(x_{0}, \kappa\right)\right)+\beta(y)-\beta\left(H\left(y, \xi_{0}\right)\right)\right)
\end{gathered}
$$

Letting $\mathrm{n} \rightarrow \infty$, we get

$\left.\mathrm{d}\left(\mathrm{H}(\mathrm{x}, \kappa), y_{0}\right)<\alpha\left(x_{0}\right)-\alpha\left(\mathrm{H}\left(x_{0}, \kappa\right)\right)+\beta(y)-\beta\left(H\left(y, \xi_{0}\right)\right)\right)$

$$
\leq \mathrm{d}\left(x_{0}, \mathrm{y}\right) \leq \mathrm{r}+\mathrm{d}\left(x_{0}, y_{0}\right)
$$

Similarly, we can prove $\mathrm{d}\left(x_{0}, \mathrm{H}(\mathrm{y}, \xi)\right) \leq \mathrm{d}\left(x, y_{0},\right) \leq \mathrm{r}+\mathrm{d}\left(x_{0}, y_{0}\right)$.

On the other hand

$\mathrm{d}\left(x_{0}, y_{0}\right)=\mathrm{d}\left(\mathrm{H}\left(x_{0}, \kappa_{0}\right), H\left(y_{0}, \xi_{0}\right)\right)$

$$
\leq \mathrm{M}\left|\kappa_{0}-\xi_{0}\right| \leq \frac{1}{M^{n-1}} \rightarrow 0 \text { as } \mathrm{n} \rightarrow \infty \text {. }
$$

So d $\left(x_{0}, y_{0}\right)=0$ implies $x_{0}=y_{0}$. Thus $\kappa=\xi$ and

$\mathrm{H}(; \kappa)=\mathrm{H}(: \xi): \overline{\mathcal{B}_{X \cup Y}}\left(x_{0}, \mathrm{r}\right) \rightarrow \overline{\mathcal{B}_{X \cup Y}}\left(x_{0}, \mathrm{r}\right)$. Thus we conclude that $\mathrm{H}(:, \kappa)$ has a fixed point in $\bar{A} \cup \bar{B}$. But this must be in A B. Therefore, all conditions of Theorem 3.2 are satisfied. Hence $\mathrm{H}($ :, $\kappa)$ has a fixed point in $\bar{A} \cap \bar{B}$. But this must be in $\mathrm{A} \cap \mathrm{B}$. Therefore, $\kappa=\xi \in \mathrm{X} \cap \mathrm{Y}$ for $\kappa \in\left(\kappa_{0}-\epsilon, \kappa_{0}+\epsilon\right) \subseteq \mathrm{X} \cap \mathrm{Y}$.

Clearly, $\mathrm{X} \cap \mathrm{Y}$ is open in $[0,1]$.

To prove the reverse, we can use the similar process.

\section{Conclusion}

In the present research, we have continued to investigate postulates of bipolar metric spaces. We have presented fixed point results by using new caristi type contractive conditions defined on bipolar metric spaces, suitable examples that supports our main results. Also, applications to integral equations as well as Homotopy theory are provided.

\section{Acknowledgement}

The authors are very thanks to the reviewers and editors for valuable comments, remarks and suggestions which improved the paper in good form

\section{References}

[1] Ali Mutlu, Utku Gürdal., Bipolar metric spaces and some fixed point theorems, J. Nonlinear Sci. Appl. 9(9), 2016, 5362-5373.

[2] Ali Mutlu, Kübra Özkan, Utku Gürdal., Coupled fixed point theorems on bipolar metric spaces, European journal of pure and applied mathematics. Vol. 10, No. 4, 2017, 655-667.

[3] J. Caristi, Fixed point Theorems for mappings satisfying inwardness condition, Trans. Amer. Math. Soc.215 (1976), 241-251. http://dx.doi.org/10.1090/s0002-9947-1976-0394329-4.
[4] Banach S., Sur les operations dans les ensembles abstraits etleur applications aux equations integrals, Fund. Math.3. 133181(1922).

[5] Farshid Khojasteh, et. al., Some applications of Caristis' fixed point theorem in metric spaces. Fixed point theory and Applications (2016), 2016:16

[6] I. Ekeland., On the variational principle, J. Math. Anal. Appl. 47(2), 324-353 (1974).

[7] I. Ekeland., Sur les problems variationnels, C. R. Acad. Sci. Paris, 275(1972), 1057-1059. 1.

[8] Lazaiz, Samih; Chaira, Karim; Aamri, Mohamed; Marhrani, EL Miloudi; Related point theorems of caristi type for two setvalued mappings, Bulletin of Mathematical Analysis and Applications. 2017, Vol. 9 Issue 1, p123-133. 11p.

[9] M. A. Khamsi, W. A. Kirk, An Introduction to metric spaces and fixed point theory, Wiley-Inter science, New York, (2001), http://dx.doi.org/10.1002/9781118033074.

[10] MA. Khamsi, Remarks on Caristis' fixed point theorem, Nonlinear Anal. TMA 71, 227-231(2009)

[11] M. R. Alfuraidan; Remarks on Caristis fixed point theorem in metric spaces with a graph, Fixed Point Theory and Applications, vol. 2014, no. 1, article no. 240, 2014

[12] RP. Agarwal, MA. Khamsi, Extension of Caristi's fixed point theorem to vector valued metric space, Nonlinear Anal. TMA 74, 141-145(2011) doi: 10.1016/ j. na.2010.08.025.

[13] Tomonari Suzuki; Characterization of -Semi completeness via Caristis Fixed Point Theorem in Semi metric Spaces, Hindawi Journal of Function Spaces. Volume 2018, Article ID 9435470 , 7 pages, https://doi.org/10.1155/2018/9435470 